\title{
Ideación suicida y autoconcepto en estudiantes mexicanos de Bachillerato.
}

\section{Suicide ideation and self-concept in mexican high school students.}

Fecha de recepción: 9-08-2019

Fecha de aceptación: 11-05-2020

\author{
Juan Francisco Caldera Montes* \\ Oscar Ulises Reynoso González* \\ Iván Alejandro Caldera Zamora* \\ Miguel Antonio Rodríguez Barba* \\ María Elena Iñiguez Gómez* \\ *Centro Universitario de Los Altos. Universidad de Guadalajara
}

\section{resumen/abstract:}

La presente investigación tuvo tres objetivos principales, estudiar la relación entre el autoconcepto y la ideación suicida en estudiantes de bachillerato, comparar ambas variables según el sexo de los alumnos e identificar la fuerza de las dimensiones del autoconcepto en la predicción de la ideación suicida. El muestreo utilizado fue no probabilístico incidental y se conformó por un total de 567 estudiantes. Se encontró una relación negativa entre la ideación suicida y los factores académico-laboral, social, familiar y físico del autoconcepto. No se encontraron diferencias entre hombres y mujeres en la ideación suicida, pero sí en los factores emocional y físico del autoconcepto. Con respecto a la predicción de la ideación, los factores "Autoconcepto familiar" y "Autoconcepto académico-laboral" conformaron el modelo, explicando entre el $12.1 \%$ y $16.2 \%$ de la varianza. Finalmente, el estudio recomienda fomentar actividades que incrementen el autoconcepto de las personas y prevenir con ello problemas de ideación.

The present research had three main objectives, to study the relationship between self-concept and suicidal ideation in high school students, to compare both variables according to the sex of the students and to identify the strength of the dimensions of self-concept in the prediction of suicidal ideation. The sampling used was incidental non-probabilistic and was formed by a total of 567 students. A negative relationship was found between suicidal ideation and the academic-labor, social, family, and physical factors of self-concept. No differences were found between men and women in suicidal ideation, but they were found in the emotional and physical factors of self-concept. With respect to the regression analysis, the factors "Family self-concept" and "Academic-labor self-concept" formed the model, which explain between $12.1 \%$ and $16.2 \%$ of the variance. Finally, the study recommends encouraging activities that increase the self-concept of people and thus prevent problems of suicidal ideation.

\section{palabras clave/keywords:}

Ideación suicida; Autoconcepto; Suicidio; Estudiantes

Suicidal ideation; Self-concept; Suicide; Students 


\section{Introcución}

La Organización Mundial de la Salud señala que alrededor de 800,000 personas mueren por suicidio cada año, además de las muchas otras que intentan quitarse la vida sin éxito, por lo cual, el suicidio es un problema de salud pública a nivel mundial (WHO/OMS, 2017). De acuerdo con los datos de la OMS, los jóvenes adultos, de entre 15 y 19 años, se consideran como uno de los grupos de edad de más alto riesgo suicida (Pianowski, Sousa de Oliveira, y Nunes, 2015). En México, durante los últimos 50 años, la tasa de suicidios ha tenido un aumento considerable, pasando de una tasa de 2.0 durante los años setenta (Saltijeral y Terroba, 1987) a una tasa de 5.7 en 2010, siendo los jóvenes de 15 a 24 años, el grupo de edad que presentó las cifras más altas (INEGI, 2011), convirtiéndose así en la tercera causa de muerte en adolescentes.

Brown, Jeglinc, Henriques y Beck (2008) apuntan que, debido a su complejidad, el estudio del suicidio se ha dividido en tres constructos teóricos: la ideación suicida, el intento suicida y el suicidio consumado. La ideación suicida, corresponde al antecedente del intento o del suicidio consumado (Vianchá, Bahamón, y Alarcón, 2013). Ésta, se define como el "pensamiento de acabar con la propia existencia" (Córdova y Rosales, 2016, p. 234), "aquellos comportamientos encubiertos dirigidos a terminar con la propia vida" (Solís, Meda, Moreno, y Palomera, 2018, p. 13), y según Beck, Kovacs y Weissman (1979), implica el deseo y la planeación de la propia muerte. Considerando entonces que la ideación o pensamiento suicida constituye un antecedente del suicidio mismo, la importancia de su estudio y comprensión es fundamental para la prevención, tanto de su intento como de su consumación.

Generalmente, el suicidio y la ideación suicida se han considerado expresiones de conflictos psicológicos que la persona experimenta por tiempos prolongados, como la depresión. Sin embargo, algunos autores como González, Díaz, Ortiz, González y González (2000), señalan que el suicidio y la ideación suicida no en todos los casos se relacionan con psicopatologías evidentes y existen casos en los que se relacionan con conflictos propios del desarrollo evolutivo. Al respecto, Borges, Rosovsky, Caballero y Gómez (1994), en concordancia con las estadísticas de la OMS, señalan que después de los 14 años la tasa de suicidios aumenta considerablemente, siendo el grupo de 20 a 24 años la punta más alta en la distribución.

Lo anterior pone de manifiesto que, para la comprensión del fenómeno suicida, es necesario considerar factores de orden biológico, social y psicológico, particularmente aquellos que tienen que ver con el desarrollo. Entre los diferentes factores asociados a la ideación suicida según la literatura especializada destacan: el abuso sexual, el consumo de drogas y alcohol, la depresión, los sentimientos de desesperanza (Toro, Grajales, y Sarmiento, 2016), el haber experimentado situaciones humillantes, la falta de apoyo social, la manifestación de conductas alimentarias de riesgo, los trastornos de conducta alimentaria y la victimización escolar (Sánchez, Villarreal, Musitu, y Martínez, 2010). También, se ha señalado relación con el apoyo familiar y el ajuste a la escuela (Reynoso, Caldera, Carreño, García, y Velázquez, 2019). Así mismo, se ha encontrado que la ideación suicida es más frecuente en mujeres que en hombres (González-Forteza, Ramos, Caballero, y Wagner, 2003). 
$\mathrm{Al}$ igual que la ideación suicida, los problemas relacionados con el autoconcepto tienen en los jóvenes uno de los grupos de edad con mayor riesgo de padecerles (Salum-Fares, Marín, y Reyes, 2011). El autoconcepto que deviene de una evaluación y percepción consciente de los individuos hacia sí mismos, (Peganos et.al., 2005), es un conjunto de imágenes, juicios y conceptos propios, acerca de distintas dimensiones psicológicas, sociales, morales y corporales (Gaeta y Cavazos, 2017). Según la postura de García y Musitu (2014), el autoconcepto se compone de cinco dimensiones fundamentales: el autoconcepto físico, que se puede entender como una autodescripción de la condición física, el atractivo y la fuerza que una persona tiene de sí misma; el autoconcepto académico o laboral, que se refiere a las autopercepciones de desempeño, ya sea comportamentales, de resultados educativos, rendimiento escolar y logros; el autoconcepto familiar, que corresponde a la percepción que tiene la persona de su implicación, participación e integración en su familia; el autoconcepto social, que implica la percepción que tienen las personas sobre su desempeño en las relaciones sociales; y el autoconcepto emocional, que constituye la percepción de los sujetos de su estado emocional y las respuestas emotivas que emiten en situaciones específicas.

Autores como Rodríguez et al. (2016) y Guedea et al. (2017) describen cómo el autoconcepto, en jóvenes universitarios y en adolescentes, se ve mayormente influido por el apoyo de las amistades y el afecto positivo (expresiones de alegría, entusiasmo, enamoramiento, etc.) en el caso de los hombres, mientras que en las mujeres las variables más relacionadas son el apoyo familiar y bajos niveles de afecto negativo (expresiones de tristeza, ira, miedo, ansiedad, etc.); por su parte, las conductas de cuidado de la salud parecen influir positivamente en el autoconcepto en los jóvenes de ambos sexos.

Asimismo, algunos estudios señalan que la relación del autoconcepto y el bienestar subjetivo en los adolescentes es esencial para un desarrollo mental saludable, ya que una autovaloración positiva de sí mismo implica niveles óptimos de satisfacción con la vida y afecto positivo, permitiendo al sujeto experimentar un mayor número de emociones positivas (Ramos et al., 2017; Rodríguez, et al., 2016). Por otro lado, Péganos et al. (2005) refieren la importancia del autoconcepto de los adolescentes en el establecimiento de relaciones románticas más positivas y en la estabilidad en las relaciones de apego, y apunta que la relación con los padres desde la infancia es un factor importante para que los adolescentes formen un modelo positivo en la percepción de sí mismos como en sus relaciones sociales.

En cuanto a los estudios que relacionan la ideación suicida y el autoconcepto en los adolescentes, Duarté, Lorenzo-Luaces, y Rosselló (2012) encontraron una correlación entre el autoconcepto negativo y los síntomas de depresión y pensamientos disfuncionales, lo cual se asocia a un menor uso de estrategias de afrontamiento y mayores niveles de ideación suicida. En otro estudio planteado por Fernández y Merito (2001), a través de un análisis de regresión múltiple, se encontró que la depresión y el autoconcepto social explican en un $51 \%$ la presencia de ideación suicida en una muestra de adolescentes, tanto en hombres como en mujeres. Finalmente cabe destacar los hallazgos de Chávez et al. (2017) y los de Duarté et al. (2012), en los que se señala la relación del autoconcepto con la ideación suicida y la sintomatología depresiva. 
En tal sentido, considerando la particularidad de estos fenómenos en la adolescencia, este estudio se planteó como objetivo analizar la relación entre la ideación suicida y el autoconcepto en la población estudiantil de Los Altos de Jalisco, México. En dicho contexto son prácticamente inexistentes las investigaciones sobre la asociación de estos dos fenómenos, mas no los datos que comprueban la ocurrencia de problemáticas al respecto (El Informador, 2010).

\section{Método}

\section{Participantes:}

Los participantes de este estudio fueron estudiantes de un bachillerato tecnológico del entorno regional de Los Altos de Jalisco, México. La muestra no probabilista e incidental se conformó por un total de 567 alumnos de los cuales 279 eran hombres y 288 mujeres. Las edades fluctuaban entre los 14 y 20 años $(M=16.29$; $D E=1.161)$. Se administró un consentimiento informado a todos los participantes. Si eran menores de edad, se solicitó el aval a sus padres o tutores. En dicho escrito se estipuló que los investigadores se comprometían a proteger los datos y solo divulgarlos con fines académicos y de manera global. Al iniciar el trabajo de campo, se informó a los respondientes acerca de los objetivos del estudio y su libertad para aceptar o rechazar su participación.

Por tales razones, el estudio se considera, según las normas éticas de investigación con seres humanos, como riesgo mínimo, garantizando los derechos y favoreciendo el bienestar de cada individuo participante. Además de ello, se les indicó a los alumnos que si la prueba les generaba algún tipo de incomodidad, podrían negarse a continuar y que el psicólogo escolar estaría disponible para dialogar si así lo quisieran.

\section{Instrumentos}

Escala de ideación suicida de Beck (ISB). Dicho instrumento fue diseñado para evaluar los pensamientos suicidas y su intensidad, además permite identificar si las personas han presentado intentos de suicidio anteriormente (Beck et al., 1979). Tiene un total de 20 ítems los cuales se valoran en una escala de 0 a 2 puntos. Si la puntuación de los reactivos 4 «Deseo de realizar un intento de suicidio activo» y 5 «Intento pasivo de suicidarse», es " 0 ", se omiten las secciones siguientes y se suspende la entrevista, codificando como: "No aplicable". De no ser así, se sigue respondiendo. La escala en un estudio previo con población mexicana obtuvo un alfa de Cronbach igual a 0.84 (González, Díaz, Ortiz, González, y González, 2000). Dentro de este estudio, tal instrumento mostró una consistencia interna adecuada ( $\alpha=$ .916).

Escala de Autoconcepto (AF-5) de García y Musitu (2014). Este cuestionario pretende evaluar la percepción que el individuo tiene de sí mismo. La prueba está constituida de 30 reactivos (p. ej. «Hago bien los trabajos escolares y/o académicos» y «Mi familia está decepcionada de mí») agrupados en cinco factores (autoconcepto académico-laboral, autoconcepto social, autoconcepto emocional, autoconcepto físico, autoconcepto familiar). El formato de respuesta es numérico y expresa el grado de acuerdo con cada reactivo (de "1" a "99"). El coeficiente de fiabilidad reportado por investigaciones anteriores ha sido de $\alpha=.73$ (Bustos, 
Oliver, y Galiana, 2015) y $\alpha=.82$ (Esnaola, Rodríguez, y Goñi, 2011). En esta investigación, tal escala obtuvo una consistencia interna adecuada $(\alpha=.912)$.

Adicionalmente, se utilizó un cuestionario de datos sociodemográficos (ad-hoc), el cual indagó sobre las variables sexo, carrera y edad de los participantes.

\section{Procedimiento}

En un primer momento, para la presentación, autorización y consentimiento del proyecto de investigación se efectuó una entrevista con las autoridades del plantel educativo. Una vez acreditado, se visitó, en sus respectivos salones de clase, a los alumnos y se solicitó que respondieran los instrumentos antes consignados. El tiempo aproximado para que los participantes respondieran las escalas fue de 25 minutos. El trabajo de campo se efectuó a finales del año 2017 y contó en todo momento con el soporte de profesores y funcionarios de la institución educativa.

\section{Análisis de datos}

Se efectuaron análisis descriptivos (medias y desviaciones estándar) y bivariados para detectar diferencias significativas entre hombres y mujeres, y correlaciones entre la ideación suicida y los factores del autoconcepto. En el caso de la comparación, se usó un conjunto de pruebas U de Mann Whitney (considerando la ausencia de normalidad en la distribución de las variables y factores) y un análisis posterior de magnitud del efecto ( $\mathrm{r}$ de Rosenthal). Para la correlación, se utilizó la prueba Rho de Spearman. Posteriormente, se realizó un análisis de regresión logística binomial mediante el método por pasos, considerando la puntuación de la prueba de "Ideación suicida" como variable dependiente (presencia-ausencia) y a los factores "Autoconcepto académico-laboral", "Autoconcepto social", "Autoconcepto familiar" y "Autoconcepto físico" como independientes. Dichos análisis se llevaron a cabo en el programa estadístico SPSS, versión 22

\section{Resultados}

De manera inicial, se calcularon las medias y desviaciones estándar de la "Ideación Suicida" y el "Autoconcepto" (incluyendo sus factores). Para su presentación, se muestran los resultados totales y por sexo (Tabla 1).

Tabla 1.- Resultados totales y por sexo de Ideación Suicida y Autoconcepto

\begin{tabular}{lcccccc}
\hline \multirow{2}{*}{\multicolumn{1}{c}{ Variable/factor }} & \multicolumn{2}{c}{ Total } & \multicolumn{2}{c}{ Mujeres } & \multicolumn{2}{c}{ Hombres } \\
\cline { 2 - 7 } & $M$ & $D E$ & $M$ & $D E$ & $M$ & $D E$ \\
Ideación suicida & 3.23 & 5.597 & 3.53 & 5.746 & 2.91 & 5.432 \\
Autoconcepto & 6.77 & 2.555 & 6.62 & 1.594 & 7.13 & 1.497 \\
Autoconcepto académico-laboral & 6.77 & 2.555 & 6.78 & 2.659 & 6.75 & 2.448 \\
Autoconcepto social & 7.26 & 1.898 & 7.17 & 1.975 & 7.36 & 1.814 \\
Autoconcepto emocional & 6.62 & 2.319 & 6.03 & 2.452 & 7.24 & 2.000 \\
Autoconcepto familiar & 8.19 & 2.117 & 8.09 & 2.249 & 8.30 & 1.969 \\
Autoconcepto físico & 5.52 & 2.471 & 5.03 & 2.534 & 6.03 & 2.302 \\
\hline
\end{tabular}

Fuente: Elaboración propia 
Posteriormente, se abordaron las diferencias entre hombres y mujeres. Los resultados se muestran en la Tabla 2.

Analizando los datos de la Tabla 2, se logran identificar diferencias significativas en el "Total de Autoconcepto" ( $\mathrm{Z}=-3.897 ; \mathrm{p}=.000)$, el "Autoconcepto Emocional" ( $\mathrm{Z}=-5.957$; $\mathrm{p}=.000)$ y el "Autoconcepto Físico" $(\mathrm{Z}=-4.782 ; \mathrm{p}=.000)$. En todos los casos los hombres

Tabla 2.- Comparación por sexo de Ideación Suicida y Autoconcepto

\begin{tabular}{lcc|c|c|c}
\hline \multirow{2}{*}{ Variable/factor } & \multicolumn{2}{c|}{ Rango promedio } & \multirow{2}{*}{$Z$} & \multirow{2}{*}{$\boldsymbol{P}$} & $\boldsymbol{r}$ \\
\cline { 2 - 3 } & Mujeres & Hombres & & & \\
\cline { 2 - 4 } Ideación suicida & 295.06 & 272.58 & -1.795 & .073 & 0.075 \\
Autoconcepto & 257.61 & 311.24 & -3.897 & .000 & 0.164 \\
Autoconcepto académico-laboral & 289.69 & 278.12 & -.841 & .400 & 0.035 \\
Autoconcepto social & 278.11 & 290.08 & -.870 & .384 & 0.037 \\
Autoconcepto emocional & 243.66 & 325.64 & -5.957 & .000 & 0.250 \\
Autoconcepto familiar & 281.47 & 286.62 & -.375 & .708 & 0.016 \\
Autoconcepto físico & 251.62 & 317.43 & -4.782 & $\mathbf{. 0 0 0}$ & 0.201 \\
\hline
\end{tabular}

Fuente: Elaboración propia

obtuvieron puntuaciones más altas que las mujeres, siendo el "Autoconcepto emocional" el factor que muestra más acentuadas tales diferencias $(r=.250)$. Sin embargo, el tamaño o magnitud del efecto en los casos antes mencionados puede considerarse como pequeño.

A continuación, se analizó la correlación entre la ideación suicida y los factores del autoconcepto (Tabla 3).

A partir de los resultados expuestos en la Tabla 3, se puede advertir que existen correlaciones negativas entre la Ideación suicida y los factores "Autoconcepto Académico-laboral", "Autoconcepto Social”, "Autoconcepto familiar" y "Autoconcepto físico", siendo la

Tabla 3.- Correlaciones entre la ideación suicida y los factores del autoconcepto

\begin{tabular}{cccccc}
\hline & $\begin{array}{c}\text { Autoconcepto } \\
\text { académico-laboral }\end{array}$ & $\begin{array}{c}\text { Autoconcepto } \\
\text { social }\end{array}$ & $\begin{array}{c}\text { Autoconcepto } \\
\text { emocional }\end{array}$ & $\begin{array}{c}\text { Autoconcep- } \\
\text { to familiar }\end{array}$ & $\begin{array}{c}\text { Autoconcepto } \\
\text { físico }\end{array}$ \\
\cline { 2 - 6 } Ideación & $-.300^{* *}$ & $-.260^{* *}$ & -.023 & $-.342^{* * *}$ & $-.245^{* * *}$ \\
Suicida & & & & & \\
\hline
\end{tabular}

Fuente: Elaboración propia

$* \mathrm{p}<.01$ 
dimensión emocional del autoconcepto la única que no mostró ningún tipo de asociación. En cuanto a la fuerza de las correlaciones, el "Autoconcepto familiar" ( $r=-.342)$ y el "Autoconcepto académico-laboral" ( $r=-.300)$ son los factores que se relacionan en mayor medida, aunque tales coeficientes podrán clasificarse como bajos.

Finalmente, se llevó a cabo un análisis de regresión logística binomial mediante el método por pasos (Wald), considerando a la "Ideación suicida" como variable dependiente, y los factores del Autoconcepto (académico-laboral, social, familiar y social) como independientes, eliminando al factor emocional al no demostrar asociación en el ejercicio anterior. Conviene referir que en tal análisis se clasificaron las puntuaciones de la escala de ideación suicida de forma dicotómica, considerando los puntajes menores a 1 como "sin ideación suicida" (55.4\%), y los mayores a 1 como “con ideación suicida” (44.6\%).

Los resultados indicaron que un segundo análisis mostró las mejores características de regresión al incluir solo dos de las variables independientes: "autoconcepto familiar" y "autoconcepto académico-laboral". Las dimensiones física y social del autoconcepto no fueron consideradas dentro del modelo.

Como se muestra en la Tabla 4, los coeficientes no estandarizados bajo signo negativo indican que la presencia de ideación suicida se acentúa cuando los niveles de "Autoconcep-

Tabla 4.- Modelo de regresión logística binomial

\section{Coeficientes no}

\begin{tabular}{lcccccc}
\multicolumn{1}{c}{ Variables/factores } & \multicolumn{2}{c}{ estandarizados } & & Wald & Sig. & Exp $(B)$ \\
\cline { 2 - 4 } & \multicolumn{1}{c}{$\boldsymbol{B}$} & Error est. & & & \\
\hline (Constante) & 2.729 & .417 & 13.446 & .000 & 15.312 \\
Autoconcepto familiar & -.228 & .056 & -7.298 & .000 & .796 \\
Autoconcepto académico-laboral & -.157 & .045 & -2.610 & .009 & .855 \\
Logaritmo de verosimilitud & 706.297 & & & & \\
$R^{2}$ de Cox y Snell & .121 & & & & \\
$R^{2}$ de Nagelkerke & .162 & & & & \\
Pronostico & $66.3 \%$ & & & & \\
\hline
\end{tabular}

Fuente: Elaboración propia

to familiar" y "Autoconcepto académico-laboral" son menores. Por otra parte, el alcance explicativo se encuentra entre los valores de Cox y Snell y Nagelkerke, es decir, que el modelo explica entre el $12.1 \%$ y el $16.2 \%$ del total de la varianza. Por otra parte, al realizar un pronóstico entre los datos observados y los valores de predicción, el modelo acierta en el $66.3 \%$ de los casos. 
Como última fase del análisis de datos, se calculó el odds ratio o razón de ventajas (OR) considerando como factor de riesgo la presencia conjunta de bajos niveles de "Autoconcepto familiar" y "Autoconcepto académico-laboral" en la ocurrencia de ideación suicida. Tal clasificación se obtuvo al establecer un punto de corte en el punto medio del rango teórico del instrumento. Se clasificó con bajo autoconcepto a los puntajes menores del punto medio de la distribución en ambos factores (familiar y académico-laboral).

Como muestra la Tabla 5, los niveles bajos de "Autoconcepto familiar" y "Autoconcepto académico-laboral" representan un factor de riesgo para la ideación suicida, ya que quienes presentan ambas condiciones de autoconcepto tienen 10.383 veces más riesgo de mostrar ideación suicida.

Tabla 5.- Tabla cruzada

\begin{tabular}{ccccc}
\hline & & \multicolumn{2}{c}{ Ideación suicida } & \\
\cline { 3 - 4 } & & Con ideación & Sin ideación & Total \\
\hline \multirow{2}{*}{ Autoconcepto } & & & 10 & 53 \\
& Con bajo Autoconcepto & 43 & 368 & 514 \\
& Sin bajo autoconcepto & 146 & 378 & 567 \\
& Total & 189 & IC (95\%) $[5.3-22.14]$ \\
\hline
\end{tabular}

Fuente: Elaboración propia

\section{Discusión}

La media encontrada en el inventario de ideación suicida $(M=3.23)$ puede considerarse alta si se toma en cuenta que el parámetro deseable del instrumento es igual a cero. Tal condición coincide con lo ya expresado en otras investigaciones (INEGI, 2011; Pianowski et al., 2015) que reportan el constante aumento de tal problemática, especialmente en la etapa de la adolescencia. Al respecto se puede afirmar que muy posiblemente ocurre debido a que, en la actualidad y especialmente en el entorno estudiado, existen condiciones desfavorables (como la falta de cohesión familiar, la violencia social - que especialmente se manifiesta en los entornos escolares-, las carencias económicas y la fuerte presión social sobre el deber ser de la juventud) que vulneran la salud mental de sus habitantes -en especial de los más jóvenes-. Tal afirmación se sustenta en información reciente que apunta altas prevalencias de suicidios consumados, el nivel socioeconómico y la incidencia de otras problemáticas en la región geográfica en donde se desarrolló el presente trabajo de indagación (INEGI, 2011).

Sobre el autoconcepto, es posible afirmar que sus niveles son también altos, en sentido positivo, (ya que el punto medio de la distribución es 5.00), siendo un poco más para los factores "Autoconcepto familiar" $(M=8.19)$ y "Autoconcepto social” ( $M=7.26)$ y más bajo, aunque también superior al punto medio, para el "Autoconcepto físico" $(M=5.52)$. Tal situación reafirma la noción de que dicha dimensión psicológica es esencial para lograr alcanzar y en su caso, continuar estudios superiores, ya que como se ha evidenciado en otras 
indagaciones, el autoconcepto relaciona de forma positiva con uno de los aspectos primordiales del contexto académico formal, a saber, el rendimiento escolar (Correa, Saldívar, y López, 2015).

En lo que respecta al hecho de no encontrar diferencias significativas en los niveles de ideación suicida entre los sexos, conviene referir que dicho hallazgo contradice lo encontrado por otras investigaciones que reportan mayores índices en el caso de las mujeres (González-Forteza et al., 2003). Dicha circunstancia puede explicarse en un primer momento, aludiendo a que las féminas que alcanzan el nivel educativo abordado han desarrollado competencias emocionales y cognitivas suficientes que las equiparan con el sexo masculino en materia de pretensiones y deseos de vivir. Sin embargo y como queda evidente con las contradicciones señaladas, dicha afirmación demanda mayores estudios al respecto.

En lo referente a que los hombres alcanzan mayores puntajes que las mujeres en el total de "Autoconcepto" y los factores "emocional" y "físico" podría argumentarse que las diferencias podrían estar asociadas a las todavía desventajas culturales en las que las mujeres se encuentran (que en ciertas ocasiones provoca falta de confianza) y a las normas de belleza por ellas interiorizadas, que, en algunos casos, pueden generar frustración y una autoconcepción más negativa acerca de su persona (González-Forteza et al., 2003).

Respecto a la correlación entre la "Ideación suicida" y el "Autoconcepto Académico laboral" (significativa y negativa) se puede argüir que el éxito en el plano académico es un factor protector frente a la ideación suicida y desde luego, uno de los riesgos en el caso de no valorarse lo suficiente en materia de logros escolares. Igualmente, la relación significativa y también negativa de la "Ideación Suicida y el "Autoconcepto Social" puede ser el resultado de vínculos interpersonales armoniosos (y por tanto de una buena concepción de uno mismo) que resultan ser protectores de las ideas y, en su caso, tentativas de privarse de la vida (Péganos et al., 2005).

Además, la correlación inversa de la "Ideación Suicida" y el "Autoconcepto Familiar", implica que la identificación positiva con el núcleo familiar, condesciende a valorar la vida de forma más favorable y por ende proteger frente a la ideación suicida (Chávez et al., 2017). Esta relación constata la evidente asociación entre ambas variables y reafirma la idea de que en las personas y especialmente en los jóvenes, la familia juega un papel fundamental en la concepción de sí mismos y en su consecuente equilibrio psicológico.

La asociación de la "Ideación suicida" y el "Autoconcepto Físico" se puede explicar citando a Santrock, (2004), quien ha señalado que en la actualidad muchos jóvenes que no logran encajar con los estándares ideales de la apariencia física (primordialmente ligadas a la delgadez) continuamente sufren de degradación en su contexto y como consecuencia, experimentan sentimientos asociados al fracaso, estrés, frustración, tristeza e incluso ideación suicida.

Finalmente, cabe señalar la primacía del "Autoconcepto Familiar" y el "Autoconcepto Académico-laboral" respecto de las otras dimensiones de autoconcepto, evidenciado por los resultados obtenidos con el análisis de regresión logística, donde, en los casos evaluados, bajos puntajes en dichas dimensiones predicen hasta en un $66.3 \%$ el tener niveles conside- 
rables de ideación suicida. Lo cual reafirma lo señalado por Ramos et al. (2017), Péganos et al. (2005) y Córdova y Rosales (2016), sobre la relación de aspectos familiares y sociales con la ideación suicida, en especial en los adolescentes.

En cuanto a limitaciones del estudio, es importante señalar que los resultados obtenidos emergen de autoinformes, situación que implica que las respuestas pueden estar sesgadas al ser el propio participante quien informa de sus conductas y actitudes. Sin embargo, a pesar de ello, es posible sugerir que en estudios posteriores se incluyan las mismas y otras variables que pudieran explicar en mayor medida el fenómeno de la ideación suicida, en especial en el contexto de los Altos de Jalisco, donde la problemática es evidente pero poco estudiada, y se confirme la importancia del autoconcepto como variable predictora. Asimismo, convendría utilizar otras estrategias de investigación, como por ejemplo modelos de ecuaciones estructurales o enfoques de investigación de corte cualitativo.

Para terminar, resta concluir que la investigación permitió evidenciar la relación entre el autoconcepto y la ideación suicida en la población adolescente. Del mismo modo, los resultados obtenidos en cuanto a los niveles de ideación suicida son alarmantes, y están en concordancia con lo referido por datos obtenidos de manera masiva y por los medios de comunicación (El Informador, 2010). De lo anterior, se concluye la importancia de seguir estudiando el fenómeno suicida, en sus diferentes acepciones, así como continuar con el escrutinio de aquellos factores que de alguna manera podrían explicarlo y por lo tanto permitir el establecimiento de estrategias de prevención más efectivas.

\section{Referencias}

Beck, A., Kovacs, M. y Weissman, A. (1979). Assessment of suicidal intention: the Scale for Suicide Ideation. Journal of Consulting and Clinical Psychology. 47(2), 343-352. doi: 10.1037/0022-006X.47.2.343

Borges, G., Rosovsky, H., Caballero, M. y Gómez, C. (1994). Evolución reciente del suicidio en México: 1970-1991. Anales, Instituto Mexicano de Psiquiatría. 5(9), 15-21. Obtenido de: http://repositorio.inprf.gob.mx/handle/123456789/6530

Brown, G., Jeglinc, E., Henriques, G. y Beck, A. (2008). Terapia cognitiva, cognición y comportamiento suicida. En Thomas E. Ellis (Dir.): Cognición y suicidio. Teoría, investigación y terapia. 51-52, México: Manual Moderno.

Bustos, V., Oliver, A., y Galiana, L. (2015). Validación del Autoconcepto Forma 5 en Universitarios Peruanos: Una Herramienta para la Psicología Positiva. Psicologia: Reflexão e Crítica, 28(4), 690-697. doi: 10.1590/1678-7153.201528406

Chavez, A., Correa, F., Klein, A., Macías, L., Cardoso, K. y Acosta, I. (2017). Sintomatología depresiva, ideación suicida y auto-concepto en una muestra de infantes mexicanos. Psicología Latinoamericana. 35(3), 501-514. doi: 10.12804/revistas.urosario.edu. co/apl/a.4733

Córdova, M. y Rosales, C. (2016). Ideación suicida: treinta años de investigación en estudiantes universitarios mexicanos. Psicología y Salud. 26(2), 233-243.

Correa, F., Saldívar, A. y López, A. (2015) Autoconcepto y estados emocionales: su relación con la motivación en adolescentes. Enseñanza e Investigación en Psicología. 20(2), 173- 183. Obtenido de: https://www.redalyc.org/pdf/292/29242799007.pdf

Duarté, Y., Lorenzo-Luaces, L., y Rosselló, J. (2012). Ideación suicida: Síntomas depresivos, pensamientos disfuncionales, autoconcepto, y estrategias de manejo en adolescentes puertorriqueños/as. Revista Puertorriqueña de Psicología. 23, 1-17. Obtenido de: https://www.redalyc.org/articulo.oa?id=233228917001

El Informador. (16 de Junio de 2010). Preocupa región Altos Sur por incremento de suicidios. Obtenido de: El Informador: https://www. informador.mx/Jalisco/Preocupa-region-Altos-Sur-por-incremento-de-suicidios-20100616-0113.html 
Esnaola, I., Rodríguez, A., y Goñi, E. (2011). Propiedades psicométricas del cuestionario de Autoconcepto AF5. Anales de Psicología, 2711), 109-117. Obtenido de: https://www.redalyc.org/html/167/16717018013/

Fernández, N., y Merito, H. (2001). Predictores de la ideación suicida: un estudio empírico en adolescentes. Revista de Psicopatología y Psicología Clínica, 6(2), 121-128. doi: 10.5944/rppc.vol.6.num.2.2001.3909

Gaeta, M. L., y Cavazos, J. (2017). Autoconcepto físico y académico en niños de contextos marginados en México. Revista Electrónica de Investigación Educativa, 192), 114-124. doi: 10.24320/redie.2017.19.2.604

García, F., y Musitu, G. (2014). AF5: Autoconcepto Forma 5. Madrid: TEA Ediciones.

González-Forteza, C., Ramos, L., Caballero, M., y Wagner, F. (2003). Correlatos psicosociales de depresión, ideación e intento suicida en adolescentes mexicanos. Psicothema, 15(4), 524-532. Obtenido de: http://www.psicothema.es/pdf/1102.pdf

González, S., Díaz, A., Ortiz, S., González, C. y González, J. (2000). Características psicométricas de la Escala de Ideación Suicida de Beck (ISB) en estudiantes universitarios de la ciudad de México. Salud Mental. 23(2), 21-30. Obtenido de: https://www. redalyc.org/pdf/582/58222304.pdf

Guedea, J., Solano, N., Blanco, J., Ceballos, 0., y Zueck, M. (2017). Autoconcepto físico, género y cuidado de la salud en universitarios mexicanos. Revista de Psicología del Deporte. 26(2), 51-59. Obtenido de: https://www.redalyc.org/ pdf/2351/235152045008.pdf

INEGI (2011). Estadísticas de intentos de suicidio y suicidios. Serie Boletín de Estadísticas, Continuas, Demográficas y Sociales. México: INEGI.

Peganos, P., Rodriguez, M., Carrillo, S., y Castro, J. (2005). Apego, relaciones románticas y autoconcepto en adolescentes bogotanos. Universitas Psychologica, 5(1), 21-36. Obtenido: https://www.redalyc.org/pdf/647/64750103.pdf

Pianowski, G., Sousa de Oliveira, E. y Nunes, M. (2015). Revisión de ideación, comportamiento suicida y medidas de evaluación psicológicas. Psicología desde el Caribe. 3211), 81-120. doi: 10.14482/psdc.32.1.5872

Ramos, E., Rodríguez, A., y Aguirre, A. (2017). El Autoconcepto y el bienestar subjetivo en función del sexo y el nivel educativo en la adolescencia. Psicología Educativa. 23(2), 89-94. doi: 10.1016/j.pse.2017.05.005

Reynoso, 0., Caldera, J., Carreño, B., García, D., y Velázquez, L. (2019). Modelo explicativo y predictivo de la ideación suicida en una muestra de bachilleres mexicanos. Psicología desde el caribe, 36(1), 82-100. Obtenido de rcientificas.uninorte.edu.co/ index.php/psicologia/article/viewFile/9399/214421443656

Rodríguez, A., Ramos, E., Ros, I., Fernández, A., y Revuelta, L. (2016). Bienestar subjetivo en la adolescencia: el papel subjetivo de la resiliencia, el autoconcepto y el apoyo social recibido. Suma Psicológica. 23(1), 1-70. doi: 10.1016/j.sumpsi.2016.02.002

Saltijeral, M. y Terroba, G. (1987). Epidemiología del suicidio y del parasuicidio en la década de 1971 a 1980 en México. Salud Pública de México. 29, 245-360. Obtenido de: http://www.saludpublica.mx/index.php/spm/article/view/250/241

Salum-Fares, A., Marín, R. y Reyes, C. (2011). Relevancia de las dimensiones del autoconcepto en estudiantes de escuelas secundarias de ciudad Victoria, Tamaulipas, México. Revista Electrónica de Psicología Iztacala, 14(2) ,255-272. Obtenido de: http://www.journals.unam.mx/index.php/repi/article/view/26037/24512

Sánchez, J., Villarreal, M., Musitu, G., y Martínez. B. (2010). Ideación Suicida en Adolescentes: Un Análisis Psicosocial. Psychosocial Intervention. 19(3), 279-287. doi: 10.5093/in2010v19n3a8

Santrock, J. (2004). Adolescencia. Psicología del desarrollo. España: McGraw Hill.

Solís, P., Meda, R., Moreno, B., y Palomera, A. (2018). Depresión e ideación suicida. Variables asociadas al riesgo y protección en universitarios mexicanos. Revista Iberoamericana de Psicología. 11(1), 12-21. Obtenido de: https://revistas.iberoamericana edu.co/index.php/ripsicologia/article/view/1293

Toro, R., Grajales, F. y Sarmiento, J. (2016). Riesgo suicida según la tríada cognitiva negativa, ideación, desesperanza y depresión. Aquichan. 16(4), 473-486. doi:10.5294/aqui.2016.16.4.6

Vianchá, M., Bahamón, M., y Alarcón, L. (2013). Variables psicosociales asociadas al intento suicida, ideación suicida y suicidio en jóvenes. Tesis Psicológica, 8(1), 112-123. Obtenido de: https://www.redalyc.org/articulo.oa?id=139029198009

WHO/OMS. (2017). Suicide. Fact sheet. Obtenido de World Health Organization: http://www.who.int/mediacentre/factsheets/fs369/ en/ 